\title{
Constitución de subjetividades en el contexto terapéutico: \\ Los discursos de la adicción en acción
}

\author{
Build up of subjectivity in therapy: \\ Discourses of addiction in action
}

Elda Alejandra Muñoz Sandoval ${ }^{1}$

\section{Resumen}

Nos proponemos reflexionar acerca del proceso de constitución de subjetividades en el contexto del tratamiento de adicciones, proceso comprendido como producto de la articulación que el sujeto en tratamiento realiza entre los discursos oficiales y sus propios discursos sobre drogas.

Desde el paradigma socioconstruccionista, específicamente la perspectiva discursiva, se analiza el efecto de discursos oficiales sobre drogas en el proceso de constitución del sujeto "paciente" durante la interacción terapéutica. El análisis demuestra que algunos discursos tienen el efecto de constituir sujetos dependientes y sin capacidad de decisión. Sin embargo, el sujeto no es un receptor pasivo de discursos, sino un agente activo que da sentido a su accionar, articulando discursos para justificar su consumo o para defender una identidad "positiva".

Palabras clave: subjetividad, discursos, drogas, socioconstruccionismo, interacción terapéutica.

1 Docente Facultad de Ciencias Sociales Universidad Central sede La Serena. Psicóloga, Magíster en Psicología Social. La Serena, Chile. E-mail: emunozs@ucentral.cl 


\begin{abstract}
We propose an analysis about the process of building subjectivities in the context of an addictions treatment. This process is a product based on the articulation developed by the subject in treatment, between official discourses and his own personal discourse about drugs.

From the socio-constructivist paradigm, this paper analyzes the effect of official discourses about drugs on the creation process of the subject called 'patient' during therapy interaction. The analysis shows that some discourses have the effect of creating dependant subjects with no abilities on taking decisions. However, the subject is not only a passive receptor of discourses, but also an active agent, giving meaning to his actions by articulating discourses to justify the drug consumption or to defend a "positive" identity.
\end{abstract}

Key words: Subjectivity, discourses, drugs, social-construction, therapy interaction.

\title{
Introducción
}

Los procesos subjetivos en ciencias sociales no son un tema reciente, sin embargo, en la actualidad una particular dimensión de dicha temática se ha instalado como una preocupación central en la reflexión y quehacer de los investigadores. Esta dimensión surge de un interesante ejercicio de autorreflexión, a saber, el rol de las propias ciencias (particularmente las ciencias sociales) en la producción de subjetividades.

Desde este marco de referencia, considerando los discursos provenientes de la disciplina psicológica, el problema que guía la presente reflexión es el proceso de constitución de subjetividad en salud mental, específicamente en el contexto de tratamiento de adicciones.

En el ámbito de la psicología de la salud se ha investigado, desde la perspectiva discursiva, acerca de la manera en que los discursos de expertos (oficiales) y las prácticas institucionales (como manejo del dolor 
y promoción en salud) construyen y posicionan sujetos (por ejemplo: en riesgo, responsable, pasivo, etc.), llegando a sugerir que dichos discursos y prácticas configuran las formas en que los sujetos experimentan su cuerpo e incluso a sí mismos (Yardley, 1979, citado en Willig, 2000).

Resultados similares se pueden apreciar en investigaciones realizadas en Chile por Grondona (1997) y Sandoval (1997), en las cuales se analizan los significados que articulan los discursos oficiales sobre consumo de drogas que circulan en los medios de comunicación social en dicho país, mostrando cómo se construye la droga como una amenaza de salud pública y al sujeto consumidor como "víctima pasiva" de dicha enfermedad.

Sin embargo, esta línea de investigación, al estar centrada exclusivamente en los discursos oficiales, no permite por sí sola aclarar del todo cómo está implicado el discurso en la constitución de subjetividad y cómo la experiencia puede ser mediada por el discurso.

Desde otra línea, Carla Willig (2000) describe investigaciones que muestran al sujeto de una manera activa frente a los discursos oficiales, es decir, aunque los sujetos se posicionen en relación a discursos expertos, no necesariamente se construyen a sí mismos de acuerdo con ellos; sino que también surgen tensiones y contradicciones debidas a los múltiples significados y discursos que coexisten en los relatos de las personas. Dichas contradicciones tienen claramente un potencial "subversivo", es decir, cada vez que los discursos oficiales están presentes en los relatos de los sujetos, también son desafiados, rechazados y criticados por los hablantes.

Finalmente, el objetivo de este artículo es reflexionar acerca del proceso de constitución de subjetividades en el contexto del tratamiento de adicciones, considerando dicho proceso como una permanente tensión entre los discursos oficiales sobre drogas y los discursos de los propios sujetos acerca de su experiencia, para lo cual se ilustra el análisis de los principales discursos oficiales (en la voz del/la terapeuta) y los discursos más relevantes acerca del consumo (en la voz de los sujetos que ingresan a un programa de tratamiento), intentado configurar cómo, a través del establecimiento de la relación terapéutica, se moviliza dicha tensión constituyéndose el sujeto como "sujeto de terapia". 


\section{Marco teórico}

\section{Desde el cognitivismo hacia la perspectiva discursiva}

El enfoque teórico asumido en la construcción del problema planteado es la perspectiva discursiva (Potter \& Wetherell, 1987), enfoque que se ha constituido en la concreción teórica y metodológica del paradigma socioconstruccionista en psicología social, por lo cual se realizará una breve referencia a dicho paradigma.

El paradigma socioconstruccionista surge en los años sesenta, influyendo fuertemente en las ciencias sociales. En psicología se constituye como una fuerte crítica al cognitivismo, en el sentido de desplazar el interés desde "aquello que nos representamos" hacia "lo que decimos" y que, como tal, es parte de la realidad social que se analiza (Crespo, 1995). Entonces el foco de interés ya no está en lo intrapersonal sino en la intersubjetividad. Según Tomás Ibáńez, esto “...no radica en las personas ni fuera de ellas, sino entre las personas, es decir, en el espacio de significados del que participan o que construyen conjuntamente" (Ibáńez, p.119, citado en Sisto, 2000) y este espacio de significados no es otra cosa que el lenguaje.

La concepción del lenguaje es pragmática y su rol se concretiza a través del concepto de discurso, el cual, siguiendo a Iñiguez y Antaki (1994), se define como "un conjunto de prácticas lingüísticas que mantienen y promueven determinadas relaciones sociales" (p. 63), así, el discurso es concebido como constitutivo de los fenómenos de los cuales da cuenta.

\section{Desde el concepto de identidad hacia el concepto de sujeto}

Según Iñiguez (2001) las nociones intrapsíquicas tradicionales ${ }^{2}$ esencializan el concepto de identidad, volviéndolo estático y descontextualizado, por lo cual propone una conceptualización de dicho fenómeno como un proceso dinámico, contextualizado y emergente desde procesos intersubjetivos. Esto supone asumir el rol del lenguaje en la constitución

2 Ińiguez realiza un análisis de las principales teorías de la personalidad y psicosociales como teoría de rasgos, psicoanálisis, teoría de rol y otras con el objetivo de demostrar la crítica aquí planteada a dichas teorías. 
tanto del concepto como de la propia experiencia de la identidad: “...es aceptar nuestra subjetividad como producida y constituida por el lenguaje (a través del discurso), por lo cual resulta difícil ubicarla en concreto en alguna 'identidad' o lugar. Se manifiesta, se forma, pero no se encuentra en si”' (Cabruja, 2000, p. 90).

Por su parte Michel Foucault (citado en Bornhauser, 2006) propone comprender el concepto de "sujeto" como un emergente desde los modos de producción de subjetividad asociados a prácticas discursivas circunscritas en momentos históricos específicos, de tal manera que el sujeto sería un efecto de la historia y del cruce de poderes que la atraviesa. El poder conforma activamente al sujeto, a través de prácticas discursivas, creando y asegurando las condiciones de existencia, pero no solo posibilita, también restringe (sujeta) al sujeto a un orden social del cual no puede escapar: el orden del discurso (Foucault, 1992).

En Foucault (citado en Bornhauser, 2006) el concepto de sujeto es un tránsito permanente desde un sujeto que es constituido, hacia uno que se constituye a sí mismo. Así, la palabra "sujeto" posee dos dimensiones: "sujeción" o sujeto sometido a otro, sumisión mediante el control y la dependencia con todos los procedimientos de individuación que el poder instala en la vida cotidiana; y "subjetivación" o sujeto adherido a su propia identidad mediante la conciencia o el conocimiento de sí, dimensión de la subjetividad que constituye la "relación consigo mismo" lo cual, según Deleuze (1987, p. 133), es "la regla facultativa del hombre libre": "En ambos casos, la palabra (sujeto) sugiere una forma de poder que subyuga a alguien y lo asujeta, lo convierte en sujeto de alguien" (Foucault, 2001, p. 1046; en Bornhauser, 2006).

En este estudio, tanto el concepto de sujeto, como la comprensión del proceso de constitución de subjetividad es afín con los desarrollos teóricos mencionados de Foucault, en el sentido de que ciertos modos de definir al sujeto se constituyen como tal en tanto argumentos que permiten justificar determinados órdenes (Sisto, 2000). Sin embargo, en la perspectiva discursiva (Potter \& Wetherell, 1987), lo que se justifica son determinadas acciones, como por ejemplo, el establecimiento de un tipo particular de relación social. 
En síntesis, se concibe la identidad como subjetividad emergente que se actualiza en las interacciones lingüísticas cotidianas en contextos específicos y se constituye a través de la articulación de discursos socialmente legitimados sobre el ser, como, por ejemplo, los discursos psicológicos.

En consecuencia, el estudio de los modos de producción de subjetividad requiere teorizar sobre cómo los discursos oficiales "cobran sentido" en el individuo, para lo cual Willig (2000) propone profundizar el estudio de la relación entre discurso, práctica, subjetividad y experiencia. Una de las respuestas a esta necesidad ha sido la teoría del posicionamiento en la cual las personas son vistas como constituidas por determinados posicionamientos en el discurso: “La 'teoría del posicionamiento' no es en ningún caso un relato unificado del ego y la entidad personal; más bien provee un conjunto de herramientas conceptuales con las cuales explorar la relación entre discurso y subjetividad” (Willig, 2000, p. 556).

\section{Sujetos al discurso: las tecnologías del yo}

Rose (1996) orienta su esfuerzo a investigar los procesos sociales a través de los cuales las personas se constituyen como sujetos de un tipo determinado, proceso que define a partir de la "amalgama" de los conceptos de Foucault citados previamente: "subjetivación” y "sujeción”, integrándolos como dos momentos de un mismo proceso: el proceso de subjetificación. Rose (1996) intenta delinear dicho proceso al caracterizar las prácticas (y sus implicancias) que se sustentan en determinados "regímenes del yo"3 y que resultan fundamentales al momento de posicionar a las personas dentro de la sociedad. Dichos regímenes “...no son neutros o sin impacto, producen sentidos del ser que pueden ser negativos, destructivos, opresivos y a la vez, sentidos con poder de cambio y liberación" (Potter \& Wetherell, 1987, p. 104). De esta forma, cada vez que se emplea una versión del ser se excluyen otras, así, la construcción de un tipo de subjetividad en el discurso, también crea una forma de sujeción: "Una subjetividad es

3 Un régimen del yo hace referencia a determinadas "versiones" o formas de conceptualización del ser que se encuentran socialmente legitimadas y que sientan las bases para determinadas acciones e intervenciones (Potter \& Wetherell, 1985). Es decir, es la manifestación de la relación poder/saber, en términos de Foucault, en la "relación consigo mismo". 
producida en el discurso como el ser es sujeto al discurso" (Parker, citado en Potter \& Wetherell, 1987, p. 109).

La preocupación de Rose (1996) por las prácticas lo ha llevado a preguntarse por los medios técnicos que se han inventado para "gobernar" al ser humano, es decir, modelar y conducir la conducta por las vías "deseables". En esta línea de análisis Foucault (1990) realiza un estudio acerca de las diferentes maneras en que se ha desarrollado (en nuestra cultura) un saber acerca del "sí mismo" y sistematizado a través de disciplinas como la psicología y la psiquiatría (entre otras). Dentro de una cierta diversidad de tecnologías, destacan las tecnologías del yo: técnicas para conducir la relación con "uno mismo" que:

...permiten a los individuos efectuar, por cuenta propia o con la ayuda de otros, cierto número de operaciones sobre su cuerpo y su alma, pensamientos, conducta, o cualquier forma de ser, obteniendo así, una transformación de sí mismos con el fin de alcanzar cierto estado de felicidad, pureza, sabiduría o inmortalidad (p. 48). Así, es esperable que los diversos regímenes del yo pongan en práctica toda la variedad de tecnologías en las diversas instituciones, en particular, en las instituciones de salud y salud mental. Según Rose (1996) las tecnologías del yo "encarnan" en prácticas y técnicas específicas que se realizan bajo la autoridad de un sistema de verdad y un "individuo autorizado", es decir, bajo las disciplinas psicológicas o psiquiátricas a través del profesional correspondiente. De esta forma, bajo la supervisión del "terapeuta", las tecnologías del yo modulan la relación consigo mismo mediante ciertas directivas como "conócete a ti mismo" o "hazte consciente de ti mismo", las cuales se "encarnan" mediante las diversas prácticas terapéuticas como la discusión grupal, la escritura de un diario o "tarea", la revelación del fuero interno, etc.

Sin embargo, en este contexto también podemos encontrar manifestaciones de resistencia a estas formas de gobierno de la conducta, es decir, el sujeto puede oponerse a las formas de individualidad que se le prescriben: Los seres humanos no son los sujetos unificados de un régimen coherente de dominación que produce personas tal como las sue- 
ña. Al contrario, viven su vida en un constante movimiento a través de diferentes prácticas que los tratan de diferentes maneras (Rose, 1996, p. 235).

\section{La producción de subjetividades en la relación terapéutica}

Diversos estudios demuestran cómo se producen subjetividades durante la interacción terapéutica, particularmente, durante las negociaciones de significado. Así, por ejemplo, Tannen y Wallat (1987, citado en Madill, Widdicombe \& Barkham, 2001) describen cómo los profesionales pueden dirigir estratégicamente la conversación terapéutica a través de la capacidad para definir tópicos sobre el tema en discusión. Por otro lado, Heritage y Sefi (1992, citado en Madill, et al., 2001) describen los medios por los cuales los "pacientes" pueden oponerse a las definiciones de los/as terapeutas, por ejemplo, a través de una forma pasiva. Según Maynard (1987, citado en Madill, et al., 2001), este tipo de interacciones competitivas o negociaciones de significados permiten que las identidades del terapeuta y del paciente emerjan durante el proceso de posicionarse en dichos roles. Las negociaciones de significados están presentes durante toda la terapia, pero resultan de especial importancia durante la definición del problema, proceso que, según los autores citados, no responde ni a la honestidad de unos ni al diagnóstico objetivo de otros, sino más bien la definición del problema sería resultado de la interacción y negociación de significados y de la importancia de las experiencias (Buttny, 1990; Hill, 1982, citados en Madill, et al., 2001).

Según Hoffman (1992), en salud mental prevalece un estilo jerárquico producto de la estructura lineal del modelo médico, en la cual el médico o terapeuta tienen el poder absoluto de diagnosticar y realizar prescripciones, con lo cual el sujeto de terapia queda en la posición de obedecer o desobedecer, pero en ningún caso queda la posibilidad de que surja, en esta relación, como sujeto responsable por su propio proceso de tratamiento.

Sin embargo, debido a que las definiciones realizadas por el/la terapeuta requieren de la aprobación del "paciente", siempre existe la posibilidad de que se produzca oposición o rechazo hacia la versión del terapeuta; 
lo cual podría ser interpretado como desobediencia o, de acuerdo a un amplio espectro conceptual, como negación, distorsiones cognitivas, etc., mecanismos que pueden servir como recursos retóricos para socavar las versiones del paciente y defender las interpretaciones terapéuticas (Schafer, 1980, citado en Madill, et al., 2001), proceso a través del cual aumenta la patologización del sujeto (Mc Namee, 1992).

En consecuencia, el tipo de relaciones que promueven y mantienen algunas prácticas en salud mental serían del tipo dominio/sumisión, por lo cual es esperable que de la relación terapéutica emerjan subjetividades del tipo obediente/desobediente, colaborador/resistente, sano/patológico, en definitiva, "paciente".

\section{Los sujetos de los discursos sobre drogas}

El tema de las drogas y su consumo es discutido desde diversos sectores de la sociedad, de aquí surge una amplia variedad de discursos que construyen el tema y, sobre todo, construyen al sujeto consumidor de drogas. Sin embargo, esta variedad de discursos no se encuentran en el mismo plano: Tomás Ibánez (1994) distingue entre "discursos de la droga" y "discursos sobre la droga”. El primero corresponde a los discursos y significados que surgen de los propios consumidores y el segundo corresponde a discursos oficiales que provienen de las diversas instituciones implicadas en el tema como salud, justicia, religión, partidos políticos, medios de comunicación, etc., de tal manera que cuando el consumidor "habla" de la droga, más bien "es hablado", es decir, es capturado por uno de los discursos sobre la droga: "En general, el discurso de la droga está controlado por el discurso sobre la droga. Entrar en la droga es dejarse capturar por uno de los discursos sobre la droga” (Ibáńez, 1994, p. 128). En este sentido, la droga "atrapa" al consumidor no solo por sus efectos fisiológicos, sino sobre todo porque el consumidor va articulando los significados culturales atribuidos a las drogas, lo que le permite dar sentido a su consumo y a su propia identidad como consumidor (Sepúlveda, Pérez \& Gainza, 1996). 
De esta forma, analizar los discursos sobre drogas significa identificar el tipo de sujeto consumidor que se está construyendo; así, la investigación realizada por Grondona (1997), en la cual se analizan los discursos oficiales sobre consumo de drogas que circulan en los medios de comunicación social en Chile, muestra cómo se construye la droga como una amenaza de salud pública y al sujeto consumidor como "víctima" pasiva de dicha enfermedad.

\section{Metodología}

Este estudio corresponde a una investigación realizada en el Programa de Tratamiento de Alcohol y Drogas del Hospital de Coquimbo, durante los años 2004 y 2005. Se optó por la metodología cualitativa debido a que el objetivo es la comprensión del proceso de constitución discursiva de subjetividad para lo cual se requiere acceder a las formas lingüísticas y estrategias retóricas con las cuales los sujetos construyen sus posicionamientos y dan sentido a su experiencia.

\section{Diseño}

El diseño corresponde a un estudio de caso, es decir, se seleccionó la Unidad de Alcohol y Drogas por ser el único programa especializado en la Región de Coquimbo que depende directamente del Ministerio de Salud, por lo cual resulta ser el caso típico ideal para comprender la constitución de identidad del sujeto que ingresa a dicho programa, considerando los discursos oficiales del Ministerio de Salud (en la voz de los/as terapeutas) en relación al tema de las adicciones.

\section{Método}

Se empleó análisis del discurso como método de investigación y como técnica de análisis de la información, tal como lo describe Iñiguez (2003), por ser la forma privilegiada de acceder a los discursos empleados en la constitución de subjetividad. 
Muestra

Se definió un muestra intencionada de tipo focalizada (Patton, 1990), es decir, se seleccionaron casos ricos en información con respecto al fenómeno de interés.

La muestra quedó constituida de la siguiente manera:

a) Equipo de profesionales del programa, en total 3 sujetos.

b) Sujetos al momento de ingresar, en la mitad y al finalizar el programa. En total 12 sujetos de ambos sexos, entre 25 y 50 años.

\section{Procedimientos de producción de información}

\section{Entrevista de ingreso}

La entrevista de ingreso se realiza al inicio del tratamiento, durante las primeras sesiones, como una forma de conocer al sujeto y evaluar tanto el motivo de consulta como su motivación al cambio. Esta información se empleó para describir las interacciones iniciales con los/as terapeutas. Para obtener un registro de esta interacción se solicitó la autorización tanto de los terapeutas como de los pacientes. Se grabaron las tres primeras sesiones de todos los pacientes ingresados voluntariamente al programa desde enero hasta mediados de marzo del año 2004.

\section{Entrevista Activa-Reflexiva}

Denzin (2001) describe la entrevista reflexiva como una técnica que difiere notoriamente de las nociones tradicionales de entrevista. Para Denzin esta modalidad no es una herramienta de obtención de información, más bien, es una estrategia que busca nuevas formas de producir información, con un enfoque reflexivo y activo.

Esta modalidad de entrevista fue empleada con todos los sujetos de tratamiento y su aplicación se realizó en dos momentos: en la mitad y al finalizar el tratamiento. Tanto las entrevistas individuales como los grupos focales fueron grabados en su totalidad, previo consentimiento de los/as informantes. 


\section{Grupo Focal}

Se define como una conversación grupal “...cuidadosamente planeada, diseñada para obtener información de un área definida de interés en un ambiente permisivo y no directivo" (Krueger, citado en García \& Rodríguez, 2000, p. 115).

Se aplicaron con el objetivo de profundizar y triangular la información obtenida en las entrevistas activo-reflexivas, por lo cual se realizaron en dos momentos de la investigación: en la mitad y al finalizar el tratamiento.

\section{Técnica de análisis de la información}

La información producida se analizó mediante el análisis de discurso tal como se comprende desde la perspectiva discursiva, específicamente se identificaron los repertorios interpretativos que se definen como: "Sistemas de términos usados recurrentemente para caracterizar y evaluar acciones, eventos y otros fenómenos" (Potter \& Wetherell, 1987, p.149).

Cabe destacar que, desde esta perspectiva, el análisis es un ejercicio de interpretación. Sin embargo, dicha interpretación no se remite solo a la comprensión hermenéutica, sino que busca una estricta captación del sentido mediante el análisis del papel del lenguaje por su uso en la relación social que se analiza (Garay, 2002).

\section{Resultados}

Los resultados serán ilustrados mediante extractos de las sesiones terapéuticas y de las entrevistas realizadas por la investigadora. Se identifican al final de la cita con la inicial del informante, la etapa en el tratamiento y el número de página de la entrevista.

\section{Repertorios empleados en el posicionamiento inicial}

Desde las primeras interacciones, tanto los/as terapeutas como los sujetos que recurren al programa de tratamiento, deben establecer sus respectivas posiciones, para lo cual usan diversas estrategias lingüísticas, posicio- 
nándose de manera tal que su voz cobre legitimidad frente al otro. Así, mediante los discursos que apelan a lo "verdadero" y a lo "real" los/as terapeutas se posicionan con el poder que les confiere la ciencia; y, a la vez, constituyen al paciente como subordinado con el deber de aceptar y obedecer. Por su parte, los sujetos se posicionan rechazando las designaciones terapéuticas, principalmente en lo referente a la definición de sí mismo como "paciente".

\section{Posicionamiento de los/as terapeutas: "Nosotros como representantes del conocimiento"}

Los/as terapeutas se posicionan como representantes de la ciencia, por lo tanto, su accionar está socialmente legitimado como lo "verdadero" y lo "real". Una de las estrategias más empleadas para construir este posicionamiento es explicitar el rol como "profesionales" y como "equipo de profesionales" durante la interacción inicial, lo que confiere el conocimiento legítimo y el poder para apoyar y enseñar a quienes carecen de él.

Así, este repertorio cumpliría la función de legitimar a los/as terapeutas y posicionarlas como representantes de la verdad, mediante la construcción de distintas posiciones de poder y de subordinación: los/as terapeutas con el poder de saber lo que le hace "bien" a las personas y los sujetos con el deber de aceptar las prescripciones terapéuticas.

De esta manera, el inicio del tratamiento puede ser interpretado como una presentación mutua donde se negocian los posicionamientos. Los/as terapeutas, desde su posición de poder-conocimiento, prescriben deberes y realidades a quienes, con menos poder, tienen el deber de obedecer y aceptar. Resta saber si el sujeto aceptará esta posición o desplegará algún tipo de estrategias para rechazarla y/o proponer otro tipo de posicionamientos.

\section{Posicionamiento de los sujetos: "Tengo un problema, pero no soy adicto"}

Es un repertorio que permite al sujeto posicionarse frente al terapeuta como necesitando ayuda, pero no para dejar de consumir, es decir, tiene un problema pero este no es el consumo de alcohol o drogas. 
Extracto 1 (M/Inicio/p.14):

1. Paciente: "Empiezo a trabajar y empiezo a recibir plata".

2. "si eso es lo que pasa que cuando tengo plata".

3. "es cuando se me descompone el cuerpo".

Aquí el problema es construido mediante la metáfora del cuerpo descompuesto a causa del dinero (líneas 2 y 3), lo cual permite al sujeto enunciante evitar el reclamo de la familia de que cada vez que recibe dinero compra droga. Su problema es algo que le ocurre en el cuerpo y la responsabilidad es del dinero.

Así, este repertorio permitiría rechazar la definición de adicto(a), atribuida a quienes asisten a este programa y, a la vez, mantenerse dentro del tratamiento ${ }^{4}$ mediante la construcción de diversos problemas para los cuales requieren ayuda.

\section{Repertorios empleados en la negociación del problema}

Desde el programa de tratamiento, el problema que afecta a sus usuarios es el consumo excesivo o indebido de alcohol y/o drogas y las consecuencias de tal comportamiento. Sin embargo, los sujetos no aceptan, inicialmente, dicha definición.

Desde esta discrepancia inicial se comprende que gran parte de los repertorios empleados por terapeutas y pacientes, durante el inicio del tratamiento, apunten a la negociación del problema mediante la aceptación o el rechazo de las propuestas de ambos actores. Así, la definición del problema implica una negociación permanente de las posiciones que se establecen desde el inicio de la interacción, de tal manera que cada aceptación/ rechazo involucra la legitimación/deslegitimación de la posición del otro.

\section{Discursos de los sujetos: "Yo controlo mi consumo"}

La construcción del consumo como un hábito que es posible cambiar a voluntad es un recurso que permite rechazar la definición del

4 La necesidad de mantenerse en el tratamiento, a pesar de rechazar el consumo como problema, aparece relacionado con las presiones familiares o laborales. 
consumo como problema y a la vez negociar la designación terapéutica de sí mismo como adicto, al posicionarse como sujetos activos y con autonomía, en definitiva, no dependientes.

Extracto $2^{5}$ (V./Inicio/p.12):

1. Terapeuta: "ya ¿y después cuál fue la otra pregunta?

2. Paciente: "no que que mis límites que sí los conozco como mamá": (.)

3. "que son diferentes límites y que todos los debería conocer y no".

4. "por ejemplo yo siento que todo es relativo también me me pasa mucho yo" (.)

5. "este este hábito de vida (.) porque siento ahora que es un hábito" (.)

6. "que se puede cambiar porque ha sido un hábito".

7. "y que lo puedo cambiar a otro hábito, digamos".

Este repertorio permitiría rechazar las definiciones dadas desde el programa y posicionarse como sujetos autónomos que controlan su comportamiento (líneas 5, 6 y 7).

Sin embargo, los sujetos están en un programa de tratamiento para adicciones, por lo tanto las terapeutas van a emplear estrategias para negociar el problema y construirlo como un sujeto con problemas de consumo, en definitiva, como "sujeto de tratamiento".

\section{Discursos terapéuticos: "La falta de conciencia"}

Como ya se mostró, el sujeto rechaza las definiciones del consumo como problema, así, el discurso de "la falta de conciencia" resulta ser una de las estrategias más empleadas por los/as terapeutas, mediante la construcción del sujeto como carente de la conciencia necesaria para darse cuenta de las consecuencias que su comportamiento tiene para sí mismo y para los demás. Esta "falta de conciencia” permite explicar por qué los "pacientes" no consideran problemático su comportamiento relacionado con el consumo de alcohol y/o drogas, por lo tanto resulta una estrategia

5 Los paréntesis indican pausas. 
retórica fundamental al momento de definir el consumo como problema y al consumidor como "adicto".

Extracto $3^{6}$ (F./Inicio/p.11):

1. Paciente: "Claro, por eso le digo yo".

2. Terapeuta: "Eso es importante ¡ah! que usted se dé cuenta también".

3. Paciente: "No, o sea".

4. Terapeuta: "De que a veces es más fácil reaccionar ante las emociones como la rabia o".

5. "como la pena o como la (.) la desesperación de repente".

6. "que ante (.) la cabeza digamos que tenemos como para razonar para darnos cuenta".

En este extracto (línea 2) el sujeto enunciante intenta establecer la necesidad de que el "paciente" se "dé cuenta" de la diferencia entre una "reacción emocional fácil" (líneas 4 y 5) y una reacción racional asociada al "darse cuenta". Al calificar esta distinción como "importante" enfatiza la naturaleza esencial de ello.

Así, este repertorio permitiría responsabilizar al sujeto sobre su comportamiento problemático tal como es definido desde el programa, es decir, tiene el efecto de posicionar (y construir) al sujeto de tratamiento como paciente-adicto (subordinado) en el contexto de interacción terapéutica, lo cual implica el deber de aceptar lo propuesto por las terapeutas (quienes poseen "la verdad").

Articulación de los discursos propios y discursos terapéuticos: "Mi problema es una enfermedad, algo que me ocurre"

A su llegada al programa, por lo general, el sujeto no cuenta con argumentaciones científicamente legitimadas que expliquen su comportamiento (explicaciones oficiales sobre el consumo), solo defiende construcciones que le permiten evadir responsabilidad y rechazar las

6 Los subrayados indican énfasis en el tono de voz. 
definiciones del consumo como problema. Sin embargo, a medida que avanza en el tratamiento ( $y$ el proceso de negociación de significados) va aceptando el posicionamiento de las terapeutas y el posicionamiento que le es conferido desde el programa mediante la articulación de los discursos oficiales, por lo cual a la mitad del tratamiento emergen repertorios en relación al consumo como un problema, es decir, como un comportamiento incorrecto, pero que queda justificado al construirlo como una enfermedad.

\section{Extracto 4 (P/Mitad/p.5):}

1. Entrevistador: “¿Consideras el alcoholismo un defecto?"

2. Informante: (0.1) "una enfermedad".

3. Entrevistador: "¿Qué significa que sea una enfermedad para ti?

4. Informante: (0.2) "Porque (0.1) es el mismo que una persona que le digan" (.)

5. "que es enferma del colon (.) y le prohíben tales y tales cosas".

6. Entrevistador: "uhum".

7. Informante: "que no las tiene que comer porque eso le afecta $(0.1)$ o una persona diabética” .

8. "que le prohíben el (.) la azúcar, en fin, le hacen una dieta especial”.

En este extracto se construye el alcoholismo como una enfermedad crónica (líneas 4, 5, 7 y 8) analogía que permite poner la agencia afuera, es decir, él/ella no decide consumir alcohol (líneas 4 y 5) como ningún diabético decide ser diabético sino que es algo que "le tocó" y tampoco decide dejar de consumir alcohol, "se lo prohíben", igual que el azúcar al diabético.

Así, los sujetos articulan el discurso terapéutico sobre el alcoholismo como enfermedad crónica, posicionándose como "buen/a paciente", sabe qué es el alcoholismo, pero no tiene ninguna responsabilidad sobre este comportamiento ni sobre la forma de cambiarlo, es decir, se posiciona como "paciente pasivo". 
En esta etapa (mitad del tratamiento) resulta llamativo cómo la articulación de los discursos terapéuticos ("enfermedad", "alcoholismo" y "adicción”) son usados para validar la externalización, es decir, dejar la responsabilidad por el comportamiento fuera de la propia decisión.

Por último, la articulación de discursos terapéuticos en esta etapa puede ser interpretada como la aceptación (total o parcial) de las definiciones dadas desde el programa, es decir, comienza la constitución del sujeto como "sujeto de tratamiento". Además, esta adscripción al programa surge en relación a otros repertorios que apuntan a la construcción de una identidad "positiva", es decir, se acepta la definición de "enfermo-adicto" pero en relación a otros discursos que permiten defender un sentido "positivo" de identidad.

Repertorios empleados por los sujetos en la construcción de una identidad positiva: "He cambiado, soy buena persona"

Considerando que el ingreso a un programa de tratamiento para adicciones implica una connotación negativa y una amenaza para la identidad, debido al estigma social y a los múltiples reproches familiares, es comprensible que los sujetos de tratamiento desarrollen a lo largo del proceso una serie de estrategias para defender una identidad "positiva". Así, emergen diversos repertorios usados por los sujetos de tratamiento para defender y mantener un sentido "positivo" de identidad, de tal manera que se construyen articulando diversos discursos en relación a valores personales, discursos terapéuticos y contraponiendo construcciones "negativas" de sí mismos (antes de su ingreso) con las identidades actuales cambiadas y "positivas" gracias al tratamiento.

\section{Extracto 5 (A/Final/p.5-6):}

1. Informante: "antes un genio pero que no no sé poh o sea no (0.1) pero como digo,"

2. "increíble me río ahora (.) pero no no me molesta hablar del pasao' ahora" 
3. "pero yo creo que al principio hablamos de no sé poh medio año o cuatro meses"

4. "desde que empezó el tema de los cuatro meses yo me costaba mucho hablar del"

5. "pasado no me gustaba que me lo tocaran" $(0.1)$

6. "pero llegó un momento en que no:: me molesta lo veo como algo que pasé"

7. "lo veo como una etapa si bien desagradable (0.1) pero en la vida una etapa que"

8. "estoy superando no quiero decir superá al tiro" (.)

9. "porque una enfermedad que uno está siempre latente"

10. "pero una enfermedad que yo controlo es una diabetes que la tengo controlá”.

Aquí el sujeto enunciante construye el problema como algo ya superado (línea 6) y lo confirma por el hecho de que ahora puede hablar de él (líneas 2, 3, 4, 5 y 6). Sin embargo, en la línea 8 cambia la descripción de "superado" a "superando" y construye su problema como una "enfermedad latente", en lo cual se aprecia una articulación de los discursos oficiales (línea 9). Sin embargo esto no amenaza su identidad, ya que se posiciona de inmediato "controlando" la cronicidad de la enfermedad, igual que cualquier enfermedad crónica (línea 10). Así, esta articulación discursiva tiene el efecto de defender su sentido de identidad al posicionarse como cambiado, con el problema controlado y con una enfermedad de la cual no es responsable.

\section{Síntesis de resultados}

Desde el inicio los/as terapeutas se posicionan como profesionales expertos/as legitimados/as desde la ciencia y el conocimiento, lo que los/ as constituye en una posición de mayor poder, el cual confiere el derecho de asignar definiciones con respecto a lo que "es el sujeto" (construcción del sujeto como "sin conciencia", "paciente"). Desde esta posición van a 
mostrar "lo correcto" apoyando y enseñando a los "pacientes" a aceptar "su problema", tal como es definido desde el programa, es decir, como una "adicción", lo cual, a su vez, los constituye como "pacientes adictos". Así, como una forma de posicionarse inicialmente en su lugar de privilegio, los/as terapeutas emplean el repertorio "Nosotros/as como representantes del conocimiento" mediante el uso de estrategias como adscribirse a las respectivas profesiones. Una vez establecido el posicionamiento inicial, los/as terapeutas deben mantenerlo durante todo el proceso, desplegando estrategias para que el sujeto "acepte" los discursos terapéuticos.

Por su parte, el posicionamiento que el sujeto "debe" asumir frente al terapeuta, aceptando las asignaciones terapéuticas, resulta particularmente sensible y amenazante para la identidad, debido a que ingresar en un programa de tratamiento para adicciones implica tener un "problema de consumo de alcohol y/o drogas", es decir, "ser adicto" o "ser alcohólico", algo que de ninguna manera configura un sentido "positivo" de identidad.

Lo anterior permite comprender la dirección de la argumentación de los sujetos frente a las designaciones terapéuticas, es decir, desde su posición de menos poder, despliegan estrategias que les permiten rechazar la definición de su consumo como problema y de sí mismo como adicto/ alcohólico. Así, mediante el repertorio "Tengo un problema, pero yo no soy adicto", se posicionan como alguien con problemas, pero construyen dichos problemas como algo diferente del consumo y, a la vez, construyen su consumo como un "hábito" controlable, negando implícitamente la designación de sí mismos como adictos.

Frente a estas construcciones los/as terapeutas despliegan diversas estrategias para posicionar al sujeto como "paciente". El repertorio "La falta de conciencia" es usado por los/as terapeutas con la función de que el sujeto acepte su consumo como problema y se defina a sí mismo como adicto/alcohólico.

Así, desde estos posicionamientos iniciales comienza el establecimiento de la relación "terapeuta"/"sujeto de terapia", relación que configura el proceso terapéutico como la negociación de significados en torno a la subjetividad de los usuarios del programa. A su vez, esta 
negociación se presenta como una disputa entre las intervenciones terapéuticas (designaciones realizadas por los/as terapeutas con respecto a lo que es/debe ser el sujeto, sus responsabilidades y derechos) y las diversas estrategias que despliega el sujeto para rechazar tales designaciones o aceptarlas parcialmente. De esta manera, la identidad que emerge durante las primeras interacciones alude a sujetos que tienen problemas de diversa índole, por lo cual necesitan permanecer en el programa. Sin embargo, no desean dejar de consumir ya que esto no es un problema, además tienen capacidad de control y decisión sobre su comportamiento, en definitiva, no son adictos.

No obstante, en la medida en que se establece la relación terapéutica surge un nuevo repertorio que configura un cambio en el posicionamiento inicial de los sujetos: "Mi problema es una enfermedad, algo que me ocurre”. Este repertorio evidencia la articulación de discursos oficiales ${ }^{7}$ y permite a los sujetos posicionarse en el programa como "paciente adicto", al construir la adicción/alcoholismo como una enfermedad de la cual no son responsables sino que, más bien, son "víctimas pasivas" mas". Así, este repertorio permite que emerja una identidad "positiva" como enfermo mediante la des-responsabilización por los problemas asociados al consumo.

De esta manera se puede inferir que durante el proceso de negociación de significados los sujetos han ido aceptando las designaciones terapéuticas en relación al consumo como problema y como enfermedad, de tal manera que en esta etapa la relación se construye sobre la base de la aceptación de la posición de privilegio del "terapeuta" (poder de designar subjetividades como "sujeto de tratamiento") y de subordinación del sujeto que ahora emerge como "sujeto de tratamiento". Además, es posible suponer que la motivación de los sujetos para aceptar las designaciones terapéuticas es la posibilidad de construir una identidad positiva a través de la relación terapéutica.

7 En el documento oficial del Ministerio de Salud (1996), se define el consumo de drogas como enfermedad.

8 Pasivos en cuanto a las causas del problema y también en cuanto a la solución del mismo. 
Por último, al finalizar el proceso se observan importantes cambios en los discursos que confieren un sentido positivo a la identidad de los sujetos. Principalmente a través del repertorio "He cambiado, soy buena persona” los sujetos articulan discursos socialmente legitimados (valóricos como "más preocupados por la familia" y terapéuticos como "con mayor conciencia”), construyéndose como cambiados y posicionándose con una identidad "positiva".

De esta manera, la construcción de una identidad positiva implica la aceptación de la definición de sí mismo como paciente y la posición de subordinación en relación con los/as terapeutas, es decir, el proceso de negociación como disputa queda limitado a espacios muy reducidos (por ejemplo, en el caso de recaídas) y la aceptación de deberes (y derechos) es máxima.

Sintetizando el proceso de construcción de la identidad de los sujetos al terminar el proceso de tratamiento, es posible sostener que se construyen como "pacientes" que tienen una enfermedad controlada, aunque latente, con muchos aspectos positivos y que han superado sus problemas, prácticamente como una persona con nueva identidad: la identidad del sujeto "enfermo" que no es responsable de los problemas asociados al consumo. Es decir, la articulación de los discursos terapéuticos permite suponer que en esta etapa se ha producido la aceptación de la posición (y la identidad) de "paciente" en el programa con un sentido de identidad "positivo" y "pasivo" frente al proceso.

\section{Discusión}

Desde una mirada psicosocial se ha estudiado la subjetividad como producto del intercambio y las relaciones sociales (específicamente la relación terapéutica), proceso fundamentalmente intersubjetivo, mediado por los regímenes del yo (Rose, 1996), de tal manera que los sujetos de tratamiento son constituidos como "pacientes-adictos" mediante los discursos oficiales ("víctima pasiva” y "sin conciencia"). Estos discursos 
posibilitan ciertas prácticas, como que los sujetos sean atendidos, pero también restringen otras, por ejemplo, anulan la posibilidad de que el sujeto sea más activo en la definición de lo que considera como su problema o de que participe en la decisión acerca del modo más adecuado de abordarlo. A su vez, la sujeción (Foucault, citado en Bornhauser, 2006) de los individuos mediante el establecimiento de una relación de subordinación entre un "individuo autorizado por la ciencia" (el profesional terapeuta) y un "individuo sin conciencia que debe" aceptar prescripciones y normas, se articula con la subjetivación, relación consigo mismo, proceso mediado por las tecnologías del yo (Foucault, 1990), que se constituyen como dispositivos de poder bajo las diversas formas en que se ejerce la intervención terapéutica, por ejemplo, técnicas y directivas relativas a los dispositivos "psi": autoconocimiento, autoconciencia, etc. Tales técnicas se concretizan mediante diversas prácticas terapéuticas como la exposición del fuero interno en terapia individual o grupal, la realización de "tareas", etc. Prácticas que finalmente promueven en el sujeto la articulación de los discursos oficiales con respecto a sí mismo, es decir, se autodefine como "sin conciencia" y "víctima de la enfermedad", discursos que lo posicionan sin responsabilidad sobre el "mal comportamiento"; pero, como ya vimos, a la vez lo inhabilitan como protagonista de su proceso en relación al consumo. Así, su ser queda "sujeto a los discursos oficiales", sujeción y subjetivación que implican atributos y también responsabilidades, de tal modo que, al construirlo de forma pasiva, lo desresponsabiliza (favoreciendo la construcción de una identidad positiva), pero a la vez lo inhabilita para ser activo en el proceso.

Pero el tratamiento no es solo "aceptación", sino más bien permanente negociación de significados a través de la cual el sujeto también se opone, en diversos grados a lo largo del proceso, a la subordinación terapéutica. Es decir, los discursos oficiales (mediante las tecnologías del yo) están en permanente conflicto con los discursos propios del sujeto (con los cuales el sujeto llega al tratamiento) de tal manera que si no se producen prácticas de sujeción y de subjetivación eficientes, predominará 
el posicionamiento del no-adicto y no-paciente, con lo cual el sujeto mantiene su comportamiento de consumo y termina por abandonar el tratamiento.

Por otro lado, el análisis muestra cómo, de forma simultánea al proceso de subjetificación, el sujeto va dando diversos sentidos a su consumo de substancias, y a su permanencia en el tratamiento, sentidos que solo pueden ser comprendidos considerando el contexto en el cual "lo dicho fue dicho". Los sentidos emergen de la articulación de diversos y contradictorios discursos en relación al consumo; incoherencias que, desde la perspectiva discursiva, no son interpretadas como errores o "disonancias cognitivas", sino como la expresión de la presencia activa del sujeto en las estructuras (comprendidas como discursos) que lo constituyen. De esta forma, se promueve la noción de un sujeto socio-histórico (coherente con la concepción de Vygotsky, 1995) que, si bien es cierto emerge a partir de lo social, es activo al momento de seleccionar discursos durante la articulación discursiva, selección guiada por la propia historia (biográfica y social) del sujeto y que finalmente le permite dar sentido a su experiencia generando una identidad positiva. Así, al inicio del tratamiento es posible encontrar relatos en los cuales se construye el consumo como "algo que no tiene nada de malo", "no es problemático"; articulados con la descripción de sí mismo como "luchando contra esto" o "conscientes de que están haciendo algo malo". En síntesis, emerge un sentido de disfrute, en relación al consumo, que solo puede lograrse con algún costo, el cual finalmente es construido como el problema que define el ingreso al programa. Durante la mitad del tratamiento surgen articulaciones entre los discursos propios y los discursos oficiales que favorecen un sentido "positivo" de identidad, articulación que permite suponer que el sujeto ha sido activo, al menos, en la selección de discursos que le permiten dar sentido a su experiencia.

De esta forma, la posibilidad de construir una identidad "positiva" supone una cierta agencia del sujeto en su proceso de subjetificación, es 
decir, se subordina, emerge como un determinado tipo de sujeto "de" los discursos, pero también "se" sujeta a ellos, generando articulaciones creativas en tanto le permitan un sentido del ser legítimo en su mundo social.

\section{Conclusión}

La producción de subjetividad en el programa de tratamiento, comprendido como un proceso social, no puede entenderse al margen de las interacciones con los/as terapeutas a lo largo del proceso, ya que es el resultado directo de la negociación permanente de significados a través de las diversas intervenciones y prácticas terapéuticas.

El análisis de los discursos oficiales realizado por Grondona (1997) permite observar cómo la psicología ha aportado con regímenes del yo (Rose, 1996) mediante el desarrollo de definiciones de lo que significa ser consumidor de alcohol o drogas: enfermo, adicto, sin conciencia, etc. A través de dichos discursos se ha legitimado la práctica de ciertas tecnologías del yo como intervenciones terapéuticas específicas, por lo cual se requiere profundizar sobre el rol de la psicología (y la psiquiatría) en la producción de prácticas lingüísticas que promueven y mantienen ciertas relaciones sociales y, sobre todo, indagar en el tipo de subjetividades que dichos discursos promueven, tanto en el ámbito de las adicciones como en psicología clínica en general.

En cuanto a los aportes de este estudio, se espera promover la reflexión al interior de los equipos que trabajan en el tema de alcohol y/o drogas, con respecto a cómo se ejerce el poder durante las interacciones, por ejemplo, cómo se acoge/rechaza al sujeto al momento de negociar la definición que el propio sujeto hace de su problema o de las formas que el sujeto considera como las más adecuadas para abordar dicho problema. Es decir, no solo se trata de promover sujetos con una identidad positiva, sino también sujetos activos y responsables por su comportamiento y su proceso terapéutico. 


\section{Referencias}

Bornhauser, N. (2006). Entre la identidad y la diferencia acerca de la lógica constitutiva de sujeto en el pensar de Jürgen Habermas y Michel Foucault. Una controversia pendiente. Rev. de Filosofía, 62, 101115. Recuperado el 16 de noviembre de 2011 de http://www.scielo. php?pid=S0718-4360200600010000\&script=sci_arttext.

Cabruja, T. (2000). Cómo construimos el mundo: relativismo, espacios de relación y narratividad. Revista Análisis, 25, 61-94.

Crespo, E. (1995). Introducción a la Psicología Social. Madrid: Universitas, S.A. Deleuze, G. (1987). Foucault. Buenos Aires: Paidós.

Denzin, N. (2001). The Reflexive Interview and a Performative Social Science. Qualitative Research, 1(1), 23-46.

Foucault, M. (1990). Tecnologías del Yo. Y otros textos afines. Barcelona: Paidós.

Foucault, M. (1992). El orden del discurso. Buenos Aires: Tusquets Editores. Garay, A. (2002). La Perspectiva Discursiva. Barcelona: Unitat de Psicología Social. Universitat Autónoma de Barcelona.

García M. \& Rodríguez M. (2000). El Grupo Focal como Técnica de Investigación Cualitativa en Salud: diseño y puesta en práctica. Revista Atención Primaria, 3(25), 181-186.

Grondona, G. (1997). Análisis de los Discursos emitido por el Gobierno, Políticos e Iglesia Católica, acerca del Consumo de Drogas. En M. Catalán (comp.). Drogas, Política y Cultura. Santiago: Programa Cono Sur-Universidad Diego Portales-Group IGIA-Comisión de las Comunidades Europeas.

Hoffman, L. (1992). Una postura reflexiva para la terapia familiar. En S. Mc Namee \& K. Gergen (Eds.) La terapia como construcción social (pp. 25-43). Barcelona: Paidós.

Ibáñez, T. (1994). Los discursos de la droga y los discursos sobre la droga. Madrid: Ilustre Colegio Nacional de Doctores y Licenciados en Ciencias Políticas y Sociología.

Ińiguez, L. (2001). Identidad: De lo personal a lo social. Un recorrido conceptual. En E. Crespo (Ed.). La Constitución Social de la Subjetividad (pp. 209-225). Madrid: Catarata.

Iñiguez, L. (2003). Análisis del Discurso. Manual para las Ciencias Sociales. Barcelona: UOC.

Iñiguez, L. \& Antaki, C. (1994). El Análisis de Discurso en Psicología Social. Boletin de Psicología, 44, 57-75. 
Mc Namee, S. (1992). Reconstrucción de la Identidad, la Construcción Comunal de la crisis. En S. Mc Namee \& K. Gergen (Eds.) (1996). La Terapia Como Construcción Social (pp. 219-232). Barcelona: Paidós.

Madill, A., Widdicombe, S. \& Barkham, M. (2001). The Potential of Conversation Analysis for Psychotherapy Research. The Counseling Psychologist, 3(29), 413-434.

Ministerio de Salud (1996). Orientaciones Técnicas para el tratamiento y Rehabilitación de Personas con Problemas de Drogas. Santiago: Publicaciones de Salud Mental, N 10.

Patton, M. (1990). Qualitative Evaluation and Research Methods. $2^{\circ}$ Edition. California: Sage.

Potter, J. \& Wetherell, M. (1987) Discourse and Social Psychology: Beyond Attitudes and Behaviour. London: Sage.

Rose, N. (1996). Identidad, Genealogía, Historia. En S. Hall \& P. Du Gay (comps.). Cuestiones de Identidad Cultural. Buenos Aires: Amorrortu.

Sandoval, J. (1997). Producción Discursiva y Problemas Sociales. El ejemplo de la construcción social del problema del consumo de pasta base. Recuperado el 13 de enero de 2008 de http://cidpa.cl/txt/7artic11.pdf.

Sepúlveda, M., Pérez, C. \& Gainza, A. (1996). El silencio de los angustiados. Revista Proposiciones, 27, 59-74.

Sisto, V. (2000). Subjetivación, Diálogos, Gritos en la Calle. Una Aproximación Heteroglósica al Estudio de la Subjetivación. Tesis para optar al grado de Magíster en Psicología Social. Barcelona: Universitat Autónoma de Barcelona.

Vygotsky, L. (1995) Pensamiento y Lenguaje. Barcelona: Paidós.

Willig, C. (2000). A Discourse-Dynamic Approach to the Study of Subjectivity in Health Psychology. Theory \& Psychology, 10, 547-570.

Fecha de recepción: 08 de agosto de 2011.

Fecha de aceptación: 06 de diciembre de 2011. 
